\title{
Alcuin et la gestion matérielle de Saint-Martin de Tours
}

\author{
Martina Hartmann
}

\section{Q OpenEdition}

\section{Journals}

\section{Édition électronique}

URL : http://journals.openedition.org/abpo/1220

DOI : $10.4000 /$ abpo. 1220

ISBN : 978-2-7535-1495-9

ISSN : 2108-6443

Éditeur

Presses universitaires de Rennes

Édition imprimée

Date de publication : 20 septembre 2004

Pagination : 91-112

ISBN : 978-2-7535-0053-2

ISSN : 0399-0826

\section{Référence électronique}

Martina Hartmann, "Alcuin et la gestion matérielle de Saint-Martin de Tours », Annales de Bretagne et des Pays de l'Ouest [En ligne], 111-3 | 2004, mis en ligne le 20 septembre 2006, consulté le 01 mai 2019. URL : http://journals.openedition.org/abpo/1220 ; DOI : 10.4000/abpo.1220 


\title{
Alcuin et la gestion matérielle de Saint-Martin de Tours
}

\author{
Martina HARTMANN \\ Docteur habilité à diriger des recherches (Privatdozentin) \\ Université de Heidelberg
}

En 796, Alcuin obtint de Charlemagne l'abbaye de Saint-Martin de Tours $^{1} ;$ ce monastère se distinguait non seulement parce qu'il contenait le tombeau de l'un des saints les plus prestigieux du royaume franc, mais également parce qu'il était une abbaye particulièrement riche ${ }^{2}$. Il est vraisemblable que par ce geste, le roi voulait à la fois récompenser son conseiller pour les services rendus, et le dédommager pour les biens qu'Alcuin avait abandonnés en Angleterre lorsqu'il s'était décidé à retourner dans le royaume franc après le meurtre d'Æthelred en $796^{3}$.

La grande importance de Saint-Martin fait parfois oublier qu'Alcuin n'était pas seulement abbé de ce monastère : il en dirigeait encore au moins cinq autres. Malheureusement, nous ne savons pas précisément quand lui furent accordés les monastères de Ferrières, dans le diocèse de Sens, ni celui de Saint-Loup de Troyes ${ }^{4}$ : on pensait autrefois qu'Alcuin les avait obtenus dès avant son dernier voyage vers l'Angleterre en 790 , mais Donald A. Bullough a signalé, dans son livre posthume ${ }^{5}$, que l'un et l'autre n'étaient attestés dans la correspondance de l'abbé qu'à partir de 798. Nous ne savons pas davantage quand l'Anglo-Saxon reçut l'abbaye de

1. Les éditeurs remercient Thomas Lienhard d'avoir traduit ce texte de l'allemand en français.

2. E. Vaucelle, La collégiale de Saint-Martin..., p. 104 sqq., et J. ChelinI, " Alcuin, Charlemagne et Saint-Martin... ", p. 19-50.

3. Cf. à ce propos F. Felten, Äbte und Laienäbte ..., p. 239.

4. Vita Alc., c. 9, p. 190 : Tempore vero aliquanto cum eo peracto, dedit [sc. Karolus Magnus] ei duo monasteria, Bethleem scilicet, quod altero nomine Ferrarias vocatur, et sancti Lupi apud Trecas.

5. D. A. Bullough, Alcuin. Achievement and Reputation, p. 342 et p. 432 sqq. : la première lettre qui range ces deux monastères parmi les abbayes d'Alcuin est la ${ }^{\circ} 153$, destinée à Arn de Salzbourg, datée d'avant la fin du mois d'août 798, p. 248, 1. 14 sqq. : et inde [c'est-à-dire de Chelles] ad Sanctum Lupum. Et ibi maxime spero me manere Septembrium mensem totum; et l. 18 sqq. : et sic Octobrio mense ad Ferrarias Sanctum Petrum visitare et ibi uet suivue ad medium illum mensem spero me esse. 
Flavigny ${ }^{6}$. En revanche, c'est en 796 qu'il nomma pour la première fois un monastère qui était probablement de petite taille, celui de Saint-Josse-surMer, non loin de la grande place commerciale de Quentovic ${ }^{7}$. De plus, le monastère de Cormery dépendait également de Saint-Martin, c'est-à-dire en l'occurrence d'Alcuin ${ }^{8}$. Enfin, Franz Felten a déduit d'une ligne dans un poème que l'abbé dirigeait aussi le petit monastère de Berge près de Liège $^{9}$. Peut-on pour autant affirmer qu'il gouvernait ainsi plus de 20000 servi, comme l'affirmait une lettre de l'évêque Elipand de Tolède, son adversaire dans la querelle de l'adoptianisme ${ }^{10}$ ? La pauvreté des sources ne permet pas de l'affirmer; mais dans tous les cas, les revenus devaient être considérables. Et comme Alcuin, si l'on en juge par sa correspondance, était loin d'être un ascète fuyant le siècle, il devait probablement apprécier grandement de pouvoir accorder avec générosité l'hospitalité ou des présents à ses nombreux élèves et amis ${ }^{11}$.

Si l'on considère la dispersion de ces abbayes sur tout l'espace franc occidental, on peut se demander comment se passait la gestion des monastères et comment Alcuin exploitait son bien et ses revenus; la question se pose notamment pour l'abbaye de Saint-Martin, qui était devenue sa résidence principale, un peu comme Michelstadt allait devenir plus tard celle d'Éginhard ${ }^{12}$. Il est vrai qu'au milieu des années 790, Alcuin, qui avait atteint la soixantaine, n'était pas particulièrement désireux de consacrer les dernières années de sa vie à la gestion d'une grande abbaye ou de quelques autres ; c'est du moins ce qu'affirme sa Vita, qui fut rédigée à Ferrières entre 823 et 829 d'après les informations rapportées par son disciple Sigulf ${ }^{13}$. Le conseiller de Charlemagne aurait plutôt souhaité se retirer à Fulda dans le monastère fondé par Boniface, et en 796, il aurait à nouveau tenté d'être

6. L'abbatiat d'Alcuin à Flavigny est attesté par la liste abbatiale de ce monastère (éd. Georg Heinrich PerTz, MGH SS 8, 1848, p. 502). Cf. également E. HLaWitschKa, "Textkritisches... ", ainsi que F. Felten, Äbte und Laienäbte..., p. 238, n.14.

7. Alc. Ep. 25, p. 66, 1. 20 sqq. : Martinus apud Sanctum Iodocum remansit infirmus. Dümmler datait cette lettre de «?794»; pour sa part, D. A. Bullough, Alcuin. Achievement and Reputation, p. 342, n. 33, proposait l'année 796 (" but almost certainly from the early part of 796 »). Cette seconde date correspond à une indication relevee par F. FELTEN, Äbte und Laienäbte..., p. 238, n. 114 : selon Sigebert de Gembloux, Saint-Josse aurait été fondée en 795. En ce qui concerne le rôle d'Alcuin comme abbé de Saint-Josse (monastère qui n'est pas nommé dans la Vita), Cf. également Alc. Ep. 232, p. 376 sqq.

8. G. DevallLy, « Cormery ». Cf. également infra, p. 103-113.

9. F. Felten, Äbte und Laienäbte..., p. 239, n. 119 : il s'agit d'un poème d'Alcuin édité par Ernst DummLeR, MGH Poet. 1, 1881, p. 249, l. 9. En revanche, F. FelTen (ibid.) rejette les hypothèses qui attribuaient à Alcuin l'abbatiat de Saint-Maximin de Trèves, ou de SainteColombe de Sens.

10. Alc. Ep. 182, p. 302. F. Felten, Äbte und Laienäbte..., p. 239, juge que cette indication est vraisemblable.

11. Alc. Ep. 150, p. 246. D'autres occurrences sont citées par F. Felten, Äbte und Laienäbte..., p. 239, n. 120.

12. À propos d'Éginhard et de sa correspondance, cf. M. STRATMANN, « Einhards letzte Lebensjahre...", p. 323 sqq.

13. En ce qui concerne la Vie d'Alcuin, on pourra se reporter à W. BERSCHIN, Biographie und Epochenstil..., p. 175-181. 
libéré de cette charge par Charlemagne : il proposait déjà la solution qui fut effectivement adoptée peu avant et après sa mort, à savoir le transfert de ses monastères à ses élèves ${ }^{14}$. Le roi des Francs parvint à convaincre Alcuin de rester à Tours en l'autorisant à confier à ses élèves les onera saeculi quam ipse habuerat, comme les appelle la Vita; et l'abbé ne se fit pas prier pour le faire ${ }^{15}$. On a voulu voir dans ces termes un devoir de présence à la cour, qui n'aurait plus été rempli par Alcuin lui-même mais par ses disciples $^{16}$; ceux-ci auraient été, si l'on croit une lettre, Fridugise, le futur chancelier de Louis le Pieux et successeur de son maître comme abbé de SaintMartin, ainsi que Wizzo-Candidus ${ }^{17}$. Mais est-ce réellement cela qui est désigné par ces onera saeculi, tellement redoutés par Alcuin que celui-ci voulut en être dispensé pour prix de sa présence à Tours?

On s'efforcera de montrer ici que cette expression désignait plutôt une éprouvante activité impliquée par cet abbatiat, celle qui consistait à administrer plusieurs monastères parfois très éloignés les uns des autres. En effet, la correspondance d'Alcuin permet d'affirmer que l'abbé, même passé la soixantaine, faisait encore le voyage pour visiter ses abbayes et effectuait parfois des séjours prolongés dans celles-ci, probablement pour vérifier que tout était en ordre. Ce fait nous est connu notamment parce qu'à plusieurs reprises, Alcuin indiqua son itinéraire à ses élèves, et notamment à Arn de Salzbourg, au cas où celui-ci aurait voulu le rencontrer ${ }^{18}$. À titre de comparaison, les lettres qu'envoya Éginhard (v. 770-840) dans les années 830 permettent de deviner que dans ses dernières années, cet autre abbé, dont le corps souffrait de multiples maux, répugnait à voyager et passait l'essentiel de son temps dans son monastère de prédilection, celui de Seligenstadt ${ }^{19}$. Il semble probable qu'Alcuin se trouvait dans une situation similaire à Tours. Dans ce cas, il aura volontiers confié à ses élèves la gestion et l'inspection de ses abbayes.

De fait, parmi les autres abbés de son époque, rares sont ceux qui sont aussi bien parvenus à imposer leurs disciples pour leur succéder dans leurs diverses abbayes ${ }^{20}$ : Fridugise ( $†$ 835), un des élèves les plus éminents, qui allait souscrire le testament de Charlemagne en 813, devint abbé de SaintMartin, puis chancelier de Louis le Pieux ${ }^{21}$; de même, l'abbaye de Ferrières fut dirigée successivement, après la mort d'Alcuin, par un des premiers disciples de celui-ci, Sigulf, puis par Adelbert et par Aldric, que la Vita cite éga-

14. Vita Alc., c. 11, p. 191 : monasteria sibi commissa suos ut inter discipulos divideret.

15. Ibid. : onera vero saeculi, quae ipse habuerat, discipulis, ut petierat, libentissime dispertiit. Fecit et ipse Albinus.

16. E. SHIPLEy DuckETT, Alcuin..., p. 278 sqq.

17. Alc. Ep. 245 (p. 393 sqq.).

18. Voir notamment Alc. Ep. 153 (citation supra, n. 5).

19. M. STRATMAnN, «Einhards letzte Lebensjahre... ", p. 334 sqq.

20. A. HAUCK, Kirchengeschichte..., p. 156 sqq.

21. Ph. Depreux, Prosopographie de l'entourage de Louis le Pieux..., p. 199, n. 11 : l'auteur rappelle que Fridugise ne fut pas le successeur immédiat d'Alcuin; cf. E. MABILLE, $L a$ Pancarte..., p. 153, ainsi qu'E. VAUCELLE, La collégiale de Saint-Martin..., p. 63 et 439. 
lement au nombre des disciples du saint ${ }^{22}$. Aldric était destiné à devenir archevêque de Sens de 821 à 836 : ce fut alors ${ }^{23}$ qu'il laissa l'établissement de Ferrières à Loup ( $†$ après 860 ), que sa correspondance allait rendre célèbre $^{24}$. De même, Apollinaire, qui succéda à Alcuin pour l'abbatiat de Flavigny, a été considéré par certains historiens comme un disciple de l'Anglo-Saxon, même s'il n'est attesté nulle part dans la correspondance de celui-ci ${ }^{25}$. Le monastère de Cormery resta sous la domination de l'abbé de Saint-Martin, c'est-à-dire de Fridugise et de ses successeurs, et Saint-Josse fut placé sous la dépendance de Ferrières, c'est-à-dire de Sigulf et de ses successeurs jusqu'à Loup. Cette énumération des abbayes qui échurent à des disciples d'Alcuin laisse penser que ces derniers s'étaient déjà vu confier la gestion de ces établissements du vivant de leur maître : Alcuin avait ainsi trouvé le moyen de se décharger de ce travail, pour consacrer davantage de temps à ses études savantes et à ses écrits. Ayant ainsi endossé d'abord les onera saeculi, c'est-à-dire la gestion temporelle, les élèves auraient ensuite obtenu l'honor, c'est-à-dire l'abbatiat, peu de temps avant ou après la mort d'Alcuin : ils auraient ainsi été bien préparés.

Les disciples que l'on vient d'évoquer n'étaient certainement pas les seuls dans ce cas, et il faut imaginer un réseau bien plus important. Toutefois, avant d'étudier la gestion des monastères et des évêchés par les élèves d'Alcuin, il convient de se demander quelles sont les sources disponibles pour savoir comment leur maître lui-même avait géré ses abbayes.

Or en ce domaine, les sources sont cruellement rares. Contrairement à son élève le plus connu, Raban Maur, qui fut abbé de Fulda puis archevêque de Mayence, Alcuin ne peut être étudié ni par un cartulaire, ni par un nécrologe de Saint-Martin, ni par un polyptyque ${ }^{26}$. Faut-il en conclure que l'AngloSaxon négligea la gestion de son monastère, parce qu'il ne pensait qu'à ses études savantes et à ses relations avec les grands esprits de son temps, ou cette absence doit-elle être imputée aux lacunes de la tradition manuscrite ${ }^{27}$ ?

Selon moi, il faut incriminer avant tout une mauvaise tradition des sources; en effet, quelques rares indices dans les textes incitent à penser qu'Alcuin s'acquitta avec soin des " devoirs domestiques " que lui imposait la gestion de ses monastères ${ }^{28}$; tel est du moins le cas si l'on prend en

22. Ph. DePreux, Prosopographie de l'entourage de Louis le Pieux..., p. 94, n. 5.

23. Il est délicat de déterminer à quelle date Aldric confia l'abbatiat à Loup : cf. Ph. Depreux, Prosopographie de l'entourage de Louis le Pieux..., p. 95, n. 15; en ce qui concerne la date de son accession à l'archiépiscopat de Sens, cf. ibid., p. 96, n. 19.

24. Ibid., p. 322 sqq. ; cf. également Ph. DePreuX, "Büchersuche und Büchertausch... ". 25. À propos d'Apollinaire, cf. E. Shipley Duckett, Alcuin..., p. 278 sqq.

26. R. KотTJE, "Schriftlichkeit im Dienst der Klosterverwaltung... "; cf. également M. StratmanN, "Schriftlichkeit... zur Zeit Ludwigs des Frommen... ", p. 93-97. Pour ce qui concerne les pertes subies par Saint-Martin lors de la Révolution française, cf. infra, p. 96, ainsi que la contribution de Mark Mersiowsky dans ce même volume, supra p. 73-80.

27. Â ce propos, cf. l'article fondamental d'A. EscH, "Überlieferungschance... ». 
compte des sources qui furent rédigées après sa mort. Ainsi, un diplôme du roi Pépin ${ }^{\text {er }}$ d'Aquitaine daté de 827 évoque plusieurs coloni de l'abbaye de Cormery venus se plaindre devant le tribunal royal : selon eux, l'abbé et l'avoué de Cormery leur imposaient des prélèvements auxquels ils n'avaient jamais été soumis, ni à leur époque ni plus tôt. Le facteur déterminant dans le règlement de ce conflit fut une descriptio de cette villa que fournirent l'abbé et l'avoué : ce texte rapportait qu'à l'époque de l'abbé Alcuin, les coloni de cette villa auraient reconnu par serment les prélèvements qui seraient imposés à chaque exploitation ${ }^{29}$. On peut en conclure qu'à l'instar de ce qui était fait également dans d'autres diocèses, Alcuin avait fait procéder à des enquêtes pour déterminer les prélèvements, et que ceux-ci ne découlaient pas du seul arbitraire du maître, mais d'une évaluation du potentiel agricole qui reposait sur un commun accord.

Mais ce diplôme révèle encore autre chose. Il est vrai que ni cette descriptio, ni celle d'une autre villa ne nous est parvenue; mais leur existence semble probable, dans la mesure où il était difficile pour Alcuin de prévoir que précisément en cet endroit, plus de vingt ans après sa mort, un conflit allait éclater à propos de ces prélèvements. De plus, en ce qui concerne Cormery, ce diplôme de Pépin n'est pas isolé : d'autres sont connus, soit parce qu'ils sont conservés, soit parce qu'ils sont attestés. Ainsi, en 800, Charlemagne effectua à Tours un séjour au cours duquel décéda son épouse Liutgarde ${ }^{30}$; il accorda alors à Alcuin que Cormery, pour deux de ses bateaux, fût exemptée de douanes ${ }^{31}$. L'abbé Fridugise obtint la confirmation de cette donation à la fois par Louis le Pieux en 808 et en $820^{32}$, et par Pépin $\mathrm{I}^{\mathrm{er}}$ d'Aquitaine, dont il fut déjà question ici, en $831^{33}$. Dans les trois cas, on invoqua en guise de précédent le diplôme de Charlemagne : celui-ci était sans doute précieusement conservé à Cormery, peut-être avec les descriptiones qui réglaient les prélèvements. Il semble même qu'il était encore présent à Cormery au XI ${ }^{\mathrm{e}}$ siècle, puisqu'à cette époque, on fut en mesure d'y concevoir un faux attribué à Charlemagne : le faussaire intégra en partie dans ce document le protocole de l'acte de 800, ainsi qu'un diplôme de Louis le Pieux en faveur de Cormery ${ }^{34}$. De même, Mabillon a encore pu voir sur

28. J'étais naguère plus pessimiste à ce propos : M. STRATMANN, " Schriftlichkeit... zur Zeit Karls des Großen ». Je reviendrai sur ce point au cours de la présente communication.

29. Léon LeVILLAIN, Recueil des actes..., n 12, p. 44 sqq. : quo continebatur quomodo sub tempus Alcuino abbate ipsi coloni ex ipsa villa [...] cum iuramento dictaveruntquod per singula mansa ex ipsa curte desolvere debebant daturum. Cf. à ce propos D. HAGERMANN, " Der Abt als Grundherr... ", ici p. 373 sqq., ainsi que, du même auteur, "Die rechtlichen Grundlagen..." ", ici p. 360 sqq.

30. $\mathrm{BM}^{2} \mathrm{n}^{\circ}$ 352c-355a. Liutgard est décédée le 4 juin $800\left(\mathrm{BM}^{2} \mathrm{n}^{\circ} 355 \mathrm{a}\right)$.

31. Diplôme de Charlemagne $\mathrm{n}^{\circ} 192$ (éd. Engelbert MuHLBACHER, MGH Diplomata Karolinorum 1, 1906, p. 25), du 2 juin $800\left(\mathrm{BM}^{2} 354\right)$; cet acte est conservé par une copie du XVI ${ }^{\mathrm{e}}$ siècle à partir du cartulaire de Cormery.

32. $\mathrm{BM}^{2}$ 518, du 7 avril 808 (éd. M. BouQuET, Recueil des historiens de la Gaule et de la France 6, Paris, rééd. 1869, n 2, p. 453), et $\mathrm{BM}^{2} 713$, du 7 mars 820 (éd. ibid., n 91 , p. 519 sqq.). 
place, vers 1700, un diplôme de l'abbé Alcuin daté de $804^{35}$, qui évoquait par la même occasion une donation foncière de Charlemagne, et qui est mentionné dans le cartulaire de Cormery; encore faut-il signaler que pour ce diplôme, l'hypothèse d'un faux ne peut être exclue ${ }^{36}$.

En ce qui concerne Saint-Martin proprement dit, les diplômes conservés sont peu évocateurs. Nous ne connaissons qu'un diplôme de Charlemagne, pour ce qui fut le monastère principal d'Alcuin : le document fut sans doute conçu vers $800^{37}$, et reprit mot pour mot les termes d'un autre acte rédigé par le même roi en faveur d'Itier ${ }^{38}$, prédécesseur d'Alcuin; on peut donc en conclure que ce premier diplôme avait été soigneusement conservé à Saint-Martin ${ }^{39}$. Il est d'ailleurs possible, comme l'a fait remarquer Donald A. Bullough, que l'abbaye n'en possédait aucun autre : dans la mesure où les sources narratives, telles que les Annales royales, n'accordent pas une grande place à Alcuin, le rôle de celui-ci à la cour de Charlemagne a peut-être été exagéré par les historiens ${ }^{40}$.

Il semble que les diplômes royaux aient encore été présents dans l'abbaye au début du XVIII ${ }^{\mathrm{e}}$ siècle : en 1711 en effet, Etienne Baluze examina les diplômes de Saint-Martin et dessina une reproduction des bulles d'or qui scellaient deux diplômes de Louis le Pieux. Puis tout fut anéanti durant la Révolution française, en même temps qu'un incendie détruisit les cartulaires du XI ${ }^{\mathrm{e}}$ et du XIII ${ }^{\mathrm{e}}$ siècle, la Pancarta nigra et la Pancarta alia de SaintMartin ${ }^{41}$.

Un autre monastère d'Alcuin, celui de Saint-Josse-sur-Mer, fournit également des preuves pour des diplômes royaux perdus : ceux-ci avaient été archivés, mais n'ont pas traversé les siècles. Loup de Ferrières, qu'on a déjà cité ici et duquel dépendait Saint-Josse, consacra presque tout son abbatiat

33. Diplôme de Pépin I ${ }^{e r}$ (cf. plus haut, n. 29) n 16, du 25 février 831. Sur l'original, on trouve la notice d'archive suivante : littera Pippini Aquitanorum regis nullius valoris ponatur in quarta cellula.

34. Diplôme de Charlemagne (cf. plus haut, n. 31) n² 259; voir à ce propos l'introduction de l'éditeur, p. 374, qui signale qu'on utilisa pour cet acte un précédent diplôme de Charlemagne (le $\mathrm{n}^{\circ}$ 192), ainsi qu'un diplôme de Louis le Pieux. Cf. également D. A. Bullough, Alcuin. Achievement and Reputation, p. 14, n. 27 : l'auteur dresse la liste complète des six diplômes royaux qui, conçus à Tours ou ailleurs, comportaient le nom d'Alcuin.

35. Le cartulaire de Cormery, éd. J.-J. BouRASSE, $n^{\circ}$ 4, p. 10-13. L'éditeur cite une remarque de Mabillon : nulla temporis nota in membrana ex qua hoc exemplum transscriptum est.

36. C'est là le point de vue de L. Levillain (cf. plus haut, n. 29) : " Dans son état actuel, l'acte est au moins suspect."

37. Diplôme de Charlemagne (cf. plus haut, n. 31) n 195, rédigé entre 796 et 800, peutêtre en 800, à l'occasion du séjour prolongé du roi à Tours : Cf. l'introduction de l'éditeur p. 261, ainsi que D. A. Bullough, Alcuin. Achievement and Reputation, p. 13 sqq., en particulier la n. 27.

38. Diplôme de Charlemagne (cf. plus haut, n. 31) n 141, rédigé en 782 en faveur d'Itier de Saint-Martin : le rapprochement est signalé par l'éditeur dans l'introduction au diplôme $\mathrm{n}^{\circ} 195$, p. 261.

39. Voir également, à ce propos, P. GASNAULT, "Les actes privés... ". 
à obtenir la restitution des biens dont le monastère avait été privé ${ }^{42}$. Sa correspondance indique qu'après la mort de Louis le Pieux, un grand du nom de Rhuodingus se serait approprié l'établissement; Loup écrivit donc à Lothaire $\mathrm{I}^{\text {er } 43}$ et à Charles le Chauve ${ }^{44}$, tout en essayant d'inciter ses amis abbés ou évêques à intervenir auprès de la cour pour plaider la cause de Saint-Josse. Déjà dans sa lettre à Lothaire $\mathrm{I}^{\mathrm{er}}$, Loup écrivait que "Charlemagne avait confié naguère la celle sancti Iudoci à Alcuin ad elemosinam exibendum peregrinis " : il s'agissait donc sans doute de prendre soin des pèlerins qui partaient des Îles britanniques en direction du continent, ce qui devait plaire tout particulièrement à Alcuin. En une autre occurrence, l'abbé de Ferrières écrivait également que Louis le Pieux, sur l'intervention de son épouse Judith, avait non seulement confirmé la donation de son père, sicut edictum illius attestatur (!), mais également concédé que les surplus qui n'auraient pas été employés pour les pèlerins devaient revenir aux moines ${ }^{45}$. Les expressions employées par Loup donnent le sentiment qu'il avait sous les yeux ces deux diplômes de Charles et de Louis, et qu'il en citait des extraits. Malheureusement, les actes ne sont pas conservés.

Comme on l'a déjà noté, ce sont là des indices indirects, provenant parfois de sources tardives, qui témoignent de la gestion de Saint-Martin et d'autres monastères d'Alcuin. Il est vrai que dans ce domaine, le rôle de l'Anglo-Saxon ne fut pas celui d'un novateur ni d'un réformateur : des listes de revenus du $\mathrm{VII}^{\mathrm{e}}$ siècle, dont certains fragments furent retrouvés récemment ${ }^{46}$, prouvent que dès l'époque mérovingienne Saint-Martin enregistrait soigneusement ses revenus, qui semblent avoir été considérables. Les diplômes royaux pour Itier, le prédécesseur d'Alcuin, confirment cette impression.

D'autres éléments plaident également pour une administration soigneuse. En particulier, Tours avait développé, avant même l'arrivée d'Alcuin mais surtout sous son abbatiat, un scriptorium de tout premier rang au sein du royaume franc ${ }^{47}$; or une telle production constituait une activité coûteuse, puisque la confection des précieux manuscrits exigeait de nombreuses peaux animales, mais également des feuilles d'or et des encres sélectionnées, ainsi que de nombreux copistes qu'il fallait nourrir.

40. D. A. Bullough, Alcuin. Achievement and Reputation, p. 13 sqq., et notamment la n. 27 : "The uniquely-influential position thus attributed to Alcuin in the circle of those around King Charles contrasts with the relative silence of genuine royal and Imperial diplomas, in which he figures only twice, as abbot of St. Martin's and probably in the same year (800)." "

41. Cf. à ce propos la contribution de Mark Mersiowsky dans le présent volume.

42. Loup de Ferrières, lettre $\mathrm{n}^{\circ} 11$ (éd. Ernst DuMmLER, MGH Ep. 4, 1902, p. 21), $\mathrm{n}^{\circ} 42$

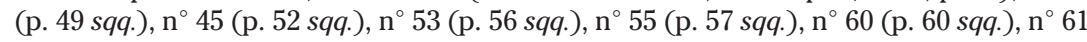
(p. $61 s q q$ ), $\mathrm{n}^{\circ} 62$ (p. $62 s q q$.), $\mathrm{n}^{\circ} 71$ (p. 67 sqq.), $\mathrm{n}^{\circ} 88$ (p. 79 sqq.) et $\mathrm{n}^{\circ} 92$ (p. 82 sqq.).

43. Ibid., lettre $n^{\circ} 11$ de 840/841, p. 21, l. 19-22: cellam sancti Iudoci, quam magnus Karolus quondam Alcuino ad elemosinam exhibendam peregrinis commiserat, beatae memoriae pater vester nobis ea ratione concessit, sicut edictum illius attestatur, ut quod elemosinae superesset in nostrum usum cederet. De même, les lettres $n^{\circ} 45,53$ et 55 mentionnent le diplôme disparu de Louis le Pieux pour Saint-Josse. 
De même, on semble avoir consigné avec précision les redevances dues par les prêtres qui, tout en se trouvant sur les terres de Saint-Martin, relevaient d'églises privées épiscopales. En effet, la collection des lettres d'Alcuin nous a transmis un écrit dans lequel l'abbé se plaignit auprès de l'évêque Raganbert de Limoges, parce que celui-ci imposait, sous peine d'interdit, que les prêtres ayant négligé de payer leur dû soient désormais soumis à des redevances plus importantes; et Alcuin en dressait la liste détaillée ${ }^{48}$. L'historiographie a accordé une attention particulière à ce passage, qui constitue une perle rare parce qu'il décrit de manière concrète les redevances que payaient les prêtres aux églises privées : en-dehors de cette source, nous n'avons aucune information précise à propos de l'importance de ces prélèvements ${ }^{49}$. Manifestement, cette augmentation des redevances inquiétait tellement les prêtres qu'ils se tournèrent vers Alcuin, bien que la cause ne relevât pas de lui; toutefois, il prit parti dans le différend. Nous ne connaissons pas l'issue de cette affaire.

Alcuin précisait que les prêtres devaient naturellement obéissance à l'évêque de Limoges, et que lui-même ferait en sorte qu'il en fût ainsi ${ }^{50}$; il s'efforçait manifestement de faire comprendre ainsi à l'évêque qu'il ne cherchait pas à s'immiscer dans les affaires de celui-ci. Il affirmait notamment l'obligation pour les prêtres d'assister au synode diocésain auprès de l'évêque dont ils relevaient. Certes, l'Anglo-Saxon ne composa jamais de capitulaire épiscopal, contrairement à son rival des dernières années de sa vie, l'évêque Théodulphe d'Orléans; mais il avait manifestement une vision très claire des devoirs à remplir par les prêtres et par les moines, ainsi que du mode de vie chrétien qu'il attribuait aux laïcs ${ }^{51}$. Et cela fut transmis à ses disciples et à d'autres personnes avec lesquelles il correspondait, parmi lesquelles des laïcs : ce dernier point est établi par nombre de ses lettres.

Mais revenons à la question posée à l'origine de cette communication, celle qui consistait à évaluer l'activité des élèves ou amis d'Alcuin dans la gestion de leurs évêchés ou de leurs monastères. Le disciple Aldric, que nous avons déjà cité, fut d'abord abbé de Ferrières puis archevêque de

44. Ibid., lettre $\mathrm{n}^{\circ} 45$ de 845, p. 52, l. 34-37 : Siquidem intercedente gloriosa matre vesra serenissimus pater vester Hludovicus Augustus ad remedium animae suae ac salutem posteritatis supplementum hoc coenobio nostro contulerat.

45. Cf. les deux notes précédentes.

46. Documents comptables de Saint-Martin de Tours...; cf. à ce propos S. SATo, "The Merovingian accounting documents...".

47. P. GaSNAUt, "Le scriptorium de Saint-Martin..." ".

48. Alc. Ep. 298, p. 456 sqq., ici p. 457, l. 9-13 : Dicunt enim : vestri missi mandassent presbiteros nostros : de pane modio I et dimidio; de vino modio I; de annona ad caballos modia quattuor; casios VI; ova $C$; pisces et orto et ligumen ad sufficientiam ab unoquoque presbitero; et si hoc non reddidissent, ex vestra auctoritate interdictum haberent missas non cantare in ecclesiis nostris nec etiam alios presbiteros in ecclesiis sancti Martini cantare licitum habere.

49. Voir à ce sujet A. HAUCK, Kirchengeschichte..., vol. 2, p. 232, n. 6, et p. 244 sqq., n. 4; cf. également M. Stratmann, "Schriftlichkeit... zur Zeit Karls des Großen... », p. 271. 
Sens jusqu'en $836^{52}$ : il encouragea la réforme monastique même après la mort de Benoît d'Aniane, réformant Saint-Amand ainsi que, de concert avec Ebbon de Reims, l'abbaye de Saint-Denis ${ }^{53}$. Un autre élève d'Alcuin, Leidrade, fut évêque de Lyon entre 797/798 et 814/816; c'était Charlemagne qui l'avait établi dans cette fonction, dans l'objectif très précis de rénover l'Eglise de Lyon qui se trouvait dans une phase de profond déclin ${ }^{54}$. Vers 813, pour rendre des comptes à l'empereur au sujet de son épiscopat, Leidrade rédigea un rapport détaillé, qui témoignait de la grande activité réformatrice de son auteur ${ }^{55}$. Cela permet de penser que c'est surtout par les efforts de celui-ci que commença le renouveau de Lyon, d'où allait émerger un important centre intellectuel sous Louis le Pieux.

On a déjà mentionné le plus célèbre des élèves d'Alcuin, Raban Maur, ainsi que les nombreux écrits qu'il a consacrés à la gestion des monastères et des évêchés ${ }^{56}$. Pour sa part, Amalaire de Metz ne s'est pas contenté d'administrer l'archevêché de Lyon lorsqu'Agobard en fut chassé, entre 835 et 838; son nom est connu avant tout par un important écrit consacré à la liturgie, ainsi que par un antiphonaire ${ }^{57}$. Arn fut abbé de Saint-Amand entre 782 et 785 , puis archevêque de Salzbourg jusqu'à sa mort en $821^{58}$. D'une part, il mit en place la bibliothèque et le scriptorium de Saint-Pierre de Salzbourg, qu'il dota de nombreux manuscrits ${ }^{59}$. D'autre part, il fit également composer une collection des lettres de son vénéré maître ${ }^{60}$ et un recueil de formulaires, ainsi que plusieurs dénombrements des possessions de l'église de Salzbourg : les Breves Notitiae et la Notitia Arnonis ${ }^{61}$. Enfin, même l'évêque Riculf de Mayence (786-813), s'il fut un élève moins illustre

50. Alc. Ep. 298, p. 457, 1. 23-26 : Quicquid enim iustum est tuam recipere ab eis auctoritatem, non renuo. Sed omnino, si tibi inoboedientes non erunt, ego tecum constringo eos. Ad tuam enim sinodum venire debent et rationem reddere de officiis spiritalibus...

51. À propos des capitulaires épiscopaux de Théodulphe (éd. Peter Brommer, MGH Capitula episcoporum 1, p. 73-142), cf. l'ouvrage à venir de R. POKORNY, Einleitung zu den Bischofskapitularien; en ce qui concerne Théodulphe de façon plus générale, on se reportera à E. DAHLHAUS-BERG, Nova antiquitas et antiqua novitas...

52. À son sujet, cf. supra, notamment les notes 23 et 24 .

53. Ph. Depreux, Prosopographie de l'entourage de Louis le Pieux..., p. 95 sqq.

54. En ce qui concerne Leidrade, cf. Ph. DePreux, Prosopographie de l'entourage de Louis le Pieux..., p. 287 sqq. ; E. Boshof, Erzbischof Agobard... ; M. STRATMAnN, "Schriftlichkeit... zur Zeit Karls des Großen... ", p. 257 sqq.

55. Rapport de Leidrade de Lyon à Charlemagne en 813/814 (éd. Ernst DuMMLER, MGH Ep. 4, 1902, n 30 , p. 542-544). Cf. à ce propos M. StRatmanN, « Schriftlichkeit... zur Zeit Karls des Großen... ", p. 257 sqq.

56. Cf. plus haut, p. 66 , et notamment la note 26.

57. A. CABANISS, Amalarius...; ;. STECK, Der Liturgiker Amalarius ...; H. SCHNEIDER, « Roman Liturgy...".

58. Pour ce qui concerne Arn, on se reportera à H. WolfRAM, Grenzen und Raüme..., p. 170-180; voir aussi, du même auteur, "Arn... ", et M. STRATMANN, " Schriftlichkeit... zur Zeit Karls des Großen... ", p. 253-256, ainsi que D. A. Bullough, Alcuin. Achievement and Reputation, passim ("Alcuin's best-documented friend in later years "). 
d'Alcuin, n'en a pas moins laissé des documents administratifs, sous la forme de litterae formatae ${ }^{62}$.

Si l'on dresse le bilan de toutes ces activités, il devient tentant de considérer que tous ces disciples avaient appris chez leur maître la nécessité, pour exercer une fonction ou pour s'élever au sein de l'Église franque, d'appliquer une gestion méticuleuse et consignée par écrit. Mais ce phénomène ne se manifeste pas seulement chez les élèves d'Alcuin : il est sensible également parmi les amis de celui-ci. Tel fut le cas d'Angilbert ( $\uparrow 814)$, qui était abbé laïc de Saint-Riquier depuis 789/790, ainsi que des frères Adalhard $(\uparrow 826)$ et Wala $(\uparrow 836)$ de Corbie. Par l'intermédiaire de la chronique d'Hariulf de Saint-Riquier, écrite au XI ${ }^{\mathrm{e}}$ siècle, nous connaissons un rapport rédigé par Angilbert ${ }^{63}$ pour rendre compte de son activité; celui-ci y décrivait la gestion et les biens dont disposait son monastère, comme l'avait fait Leidrade à propos de Lyon : il indiquait le nombre des manuscrits dans la bibliothèque, les autels, les reliques et les autres pièces du trésor ${ }^{64}$. De plus, Angilbert composa une institutio de diversitate officiorum qui décrivait les devoirs des membres du monastère ${ }^{65}$.

Pour sa part, Adalhard ${ }^{66}$ était un cousin de Charlemagne, et fut abbé de Corbie depuis 780 jusqu'à sa mort en 826 , avec une interruption de sept ans durant laquelle il fut banni par Louis le Pieux. Vers 821/822, il composa des instructions de gestion pour son monastère : selon Brigitte Kasten, ce document « constitue la source la plus détaillée pour l'organisation économique qui régnait au sein d'un monastère carolingien ", et prouvait le " talent d'organisation " d'Adalhard ${ }^{67}$. Celui-ci mort, ce fut son frère Wala, de vingt ans

59. Les manuscrits qui restent conservés sont répertoriés par G. MoSER-MERSKY et M. Minaliuk, Mittelalterliche Bibliothekskataloge..., p. 10 sqq.; on pourra y ajouter R. POKORNY, "Ein unbekannter Synodalsermo..." ".

60. Il s'agit des manuscrits 795 et 808 de la Bibliothèque Nationale de Vienne en Autriche. L'ouvrage de D. A. Bullough, Alcuin. Achievement and Reputation, p. 44-57, fait désormais référence à ce propos. Le manuscrit 795 a donné lieu à un fac-similé : F. UNTERKIRCHNER, Codex Vindobonensis 795...

61. Pour ce qui concerne les formulaires, cf. B. BischOFF, Salzburger Formelbücher...; pour les dénombrements, voir F. LOŠEK, "Notitia Arnonis und Breves Notitiae... ".

62. Pour ce qui est de Riculf lui-même, voir M. STRATMAnN, "Schriftlichkeit... zur Zeit Karls des Großen... ", p. 252 sqq., ainsi que D. A. Bullough, Alcuin. Achievement and Reputation, p. 358 et p. 362 sqq. ; quant aux litterae formatae, on pourra les trouver dans les MGH Leges 5 : Formulae, éd. Karl ZEumer, n ${ }^{\circ}$ 13, p. 559, ainsi que dans les Chartae Latinae Antiquiores 2, éd. Albert BRUCKNER - Robert MARICHAL, n 176, p. 133. En ce qui concerne la deuxième lettre, voir également M. MERsiowsky, " Regierungspraxis und Schriftlichkeit...", p. 121.

63. L'historiographie consacrée à Angilbert fut présentée par M. STRATMANN, "Schriftlichkeit... zur Zeit Karls des Großen... ", p. 264-266; cf. également S. VIARRE, "Un portrait d'Angilbert... "; R. POKORNY, "Eine Brief-Instruktion... "; ou encore D. A. Bullough, Alcuin. Achievement and Reputation, passim.

64. Hariulf de Saint-Riquier, Chronique de l'abbaye de Saint-Riquier, p. 57-69. L'auteur et cette source, ainsi que la littérature qui leur fut consacrée, sont présentés par Pierre BOURGAIN, "Hariulf..." ".

65. Éd. Kassius HALLINGER, Siegburg (Corpus consuetudinum Monasticarum 1), 1963, p. 283-303. 
son cadet ${ }^{68}$, qui lui succéda ${ }^{69}$. Cet abbatiat fut pourtant interrompu rapidement, car Wala tomba à son tour en disgrâce auprès de Louis le Pieux et partit pour l'Italie. Il y dirigea le monastère de Bobbio jusqu'à sa mort en 836, et ce fut là qu'il rédigea un bref encore plus précisément consacré aux questions économiques et administratives que ne l'étaient les statuts de son frère ${ }^{70}$. Ce texte, il est vrai, n'est conservé que sous une forme fragmentaire; il permet pourtant de percevoir que Wala souhaitait améliorer l'organisation et la gestion de son monastère, et fixer cela par écrit à l'intention des membres de sa communauté mais aussi des générations futures.

J'en viens à ma conclusion. De toute évidence, l'élite spirituelle du royaume franc à l'époque de Charlemagne était bien consciente que pour construire l'Église franque, il ne suffisait pas de produire des ouvrages liturgiques unificateurs ou des préceptes normatifs destinés à régler la vie des clercs et des moines : il fallait également faire le compte des biens matériels des églises et des monastères, afin de conserver ces ressources et d'exploiter cette richesse pour faire croître l'Église franque. Dans ce domaine, le rôle d'Alcuin semble avoir été considérable, même si on ne peut invoquer pour cela que des indices indirects en raison de la mauvaise tradition des sources. Les rares textes conservés, ainsi que le rôle joué par les amis ou élèves de l'Anglo-Saxon, prouvent bien que dans les années qui suivirent la mort de ce dernier, la semence produisit du fruit : si l'Église franque s'épanouit alors, ce fut aussi parce qu'elle était mieux équipée dans le domaine matériel. Peut-être pourrait-on aller plus loin : si Charlemagne puis Louis le Pieux s'efforcèrent de protéger les biens d'Église et de regagner les biens qui avaient été aliénés, n'était-ce pas parce que ces initiatives avaient trouvé un puissant moteur en la personne d'Alcuin? Celui-ci avait en effet eu la possibilité de découvrir, à Tours, des structures de gestion qui, depuis l'époque mérovingienne, avaient été maintenues à travers les siècles ${ }^{71}$.

66. À son sujet, cf. B. KASTEN, Adalhard..., passim; M. STRATMANN, « Schriftlichkeit... zur Zeit Ludwigs des Frommen... ", p. 89-92; et Ph. DePREux, Prosopographie de l'entourage de Louis le Pieux..., p. 76-79.

67. Telle est l'opinion de B. KASTEN, Adalhard..., p. 174. Les Statuta seu Brevia Adalhardi abbatis Corbeiensis et les Capitula domni Adalhardi abbatis de admonitionibus in congregatione sont édités par Josef SEMmLer, Siegburg, Schmitt (Corpus Consuetudinum Monasticarum 1), 1963, p. 357-408 et p. 408-418. On consultera également avec profit A. E. Verhulst, J. SEMMLER, "Les statuts d'Adalhard... ", ainsi que B. KASTEN, Adalhard..., p. 110 sqq., ainsi que Fred ScHWIND, « Zu karolingerzeitlichen Klöstern... ».

68. À propos de Wala, cf. L. WeinRich, Wala ...; M. Stratmann, « Schriftlichkeit... zur Zeit Ludwigs des Frommen... ", p. 92 sqq.; et Ph. Depreux, Prosopographie de l'entourage de Louis le Pieux..., p. 390-393.

69. Cf. à ce sujet K. H. KRUGER, "Zur Nachfolgeregelung von 826... ». 


\section{RESUME}

Avec Saint-Martin de Tours, Alcuin reçoit de Charlemagne non seulement une abbaye très riche, mais aussi très importante à cause de saint Martin, un des plus fameux saints du monde carolingien. Mais Alcuin reçoit également au moins cinq autres abbayes comme Ferrières, Flavigny, Saint-Loup de Troyes, Saint-Josse-sur-Mer, ainsi que Berge située près de Liège. Il a visité ces monastères régulièrement et en a probablement contrôlé la gestion. Malheureusement, à ce sujet, nous n'avons pas de sources de l'époque d'Alcuin, mais des sources plus tardives. Nous savons en outre que ses amis à la cour de Charlemagne, comme Angilbert de Saint-Riquier ou Adalhard et Wala de Corbie par exemple, et ses élèves comme Arn de Salzbourg, Leidrade de Lyon ou Raban Maur ont instauré dans leurs diocèses ou monastères une administration qui recourait à l'écrit. On propose ici d'admettre que c'était Alcuin qui leur avait enseigné cela et qui avait eu une certain influence sur Charlemagne en ce sens.

\section{ABSTRACT}

With Saint-Martin in Tours Alcuin received form Charlemagne not only a very rich abbey but also a very famous one, because saint Martin had been one of the most popular saints of the Carolingian world since Merovingian times. It is because of this prominent and wealthy monastery that we forget very easily that Alcuin also received from the king not less than five other abbeys, namely Ferrières, Flavigny, Saint-Josse-sur-Mer, Saint-Loup in Troyes and Berge near Liège. Alcuin visited them all from time to time in order to control whether the administration and the religious life were in order. Unfortunately we have very few sources from Alcuins lifetime about his administration of all his monasteries, but we can see from later sources that he did his "home work" in administrating these abbeys. We can also see this by analysing the activities of his scholars in these fields like bishop Arn of Salzburg, bishop Leidrad of Lyon or Hrabanus Maurus for example, or of his friends at the court like Angilbert of Saint-Riquier or Adalhard and Wala of Corbie. Perhaps Alcuin exercised a special influence on Charlemagne in showing him how to establish a well-working administration in every church and every monastery of his regnum in order to support the Carolingian Renaissance, which required material sources.

70. Breve memorationis Walae abbatis a. 834/36 Bobbiensi monasterio datum, éd. Josef SEMmLER, Siegburg (Corpus Consuetudinum Monasticarum 1), 1963, p. 420 sqq. ; cf. également L. WeINRICH, Wala..., p. 86 sqq. 